\title{
Professional Education of People with Disabilities in a Digital Environment
}

\author{
Alexander G. Stanevskiy ${ }^{1 *}$ and Lyubov P. Khrapylina ${ }^{2}$ \\ ${ }^{1}$ Bauman Moscow State Technical University, 2nd Baumanskaya str., 5/1, 105005, Moscow, Russia \\ ${ }^{2}$ IPACS RANEPA, prosp. Vernadskogo, 84, 119606, Moscow, Russia
}

\begin{abstract}
The article informs the conceptual approaches to the professional education of people with disabilities in the digital environment and the experience of their practical implementation in high school technical education on the example of people with hearing impairments. The characteristic of individual technologies using which a digital environment is created, focused on such students is given. The technological and organizational basis for the inclusion of persons with disabilities in the educational process in the designated environment is presented, taking into account the specifics of the educational and rehabilitation needs of different categories of people with hearing impairments.
\end{abstract}

\section{Introduction}

Digital education is becoming an integral part of vocational education focused on the knowledge economy. In several countries, experience has been accumulated that confirms the progressive role of such education in the training of demanded qualified specialists. $[1,2]$. Specialists of science and practice are applying more and more importance to digital technologies as applied to the vocational education of disabled people, believing that these technologies will significantly increase the students 'acquisition of new knowledge, skills, and abilities as applied to their professional activities [4,5]. However, the experience of digital education of persons with disabilities has not been sufficiently studied and there is very little information about this. All this served as the basis for the present study, the results of which are presented in this article.

\section{Research methodology}

The basis for the study was the Head Training Center for the Professional Rehabilitation of Persons with Disabilities of Health (Disabled) of Bauman Moscow State Technical University (research university), in which, since 1934, people with hearing impairment receive professional engineering education. The Center has gained great experience in such education, special technologies have been developed, an educational, rehabilitation space, and an inclusive environment have been created, and digital technologies are being actively

* Corresponding author: $\underline{\text { stan@ @mstu.ru }}$ 
introduced. Achievements of the Center are confirmed by the fact that all graduates from the number of persons with disabilities of this university after completion of training are successfully employed and have stable employment.

In the process of this study, the results of the practical application of digital technologies in the vocational education of 875 hearing impairments who studied at the university over the past 5 years, and the features of creating a digital environment for the benefit of these students at the university were analyzed. This allowed us to formulate the following conceptual, technological and organizational approaches to the professional education of persons with disabilities in the digital environment.

\section{Conceptual approaches to the vocational education of disabled people in a digital research environment}

The concept underlying the study is the development and application of cognitive technological innovations [11,12,13], which identify and remove barriers, providing information perception for people with sensory impairments (hearing), in order to ensure their more full participation in society, in education and in the workplace, thereby increasing their productivity and the quality of their life.

The long-term (85 years) successful practice of teaching a large contingent of hard-ofhearing students gives grounds to assert that the essence and core of the educational (re) habilitation process should be the creation of such an educational environment in which the mechanisms of auditory-speech and auditory-visual perception are formed oral and written speech, which is the basis for the further development of cognitive functional systems. The development (addition) of cognitive function and the correct speech environment changes the paradigm of teaching a deaf student in inclusive conditions.

Digital education is the general name for digital technologies used in education. The digitalization of vocational education for persons with disabilities should be interpreted as a transition to the practice of providing educational services using ICT (information and communication technologies) and to new ways of cooperation (including speech) of a student with disabilities with all subjects of the educational process (teachers, staff, professionals and valid students). The very nature of ICT makes it possible to formulate individual cognitive strategies corresponding to special educational needs. The targeted use of digital technologies can increase efficiency and improve the educational process in its inclusive form. This can bring significant advantages in terms of improving the educational process and increasing its effectiveness, including with regard to achieving a rehabilitation socio-economic effect, which is primarily manifested in the success of the labor activity of graduates of professional educational institutions.

We propose to consider the digital educational environment as an environment adapted to the special educational needs of a disabled person in an educational institution, providing him with the conditions for the proper development of professional educational programs and the formation of specialist competencies, the development of general culture, adaptation to public life, and mastery of ways to resolve issues in different areas of life. This environment should have the properties of a rehabilitation effect, contribute to minimizing the disability of a disabled person in obtaining such a professional education that allows him to speak on the general labor market on an equal competitive basis with other specialists and reduces the risks of not being allowed to work. Such restrictions include restrictions on communication, orientation, training, employment, and the practically deaf in some aspects of self-care. We especially emphasize the limitations of life for this category of students regarding access to information and communication, and restrictions on the adequate perception of available information. 
Designing a digital educational environment, taking into account the above features of persons with disabilities, should be focused on:

- improvement of the technologies of vocational education already used in practice and newly introduced;

- the possibility of systematic integration of technical means of training and rehabilitation of the new digital generation with elements of artificial intelligence;

- formation of a barrier-free environment for the hearing impaired;

- ensuring the health of persons with disabilities;

- the implementation of the staged personified rehabilitation and socio-psychological support of the educational process;

- creation of conditions for the realization of potential personality abilities.

It should be borne in mind that the formation of this environment involves the creation of an integrated system that focuses on the interests of the entire contingent of persons with disabilities, and within systems - subsystems that reflect the interests of certain categories of people with disabilities, as well as individuals.

The digital educational environment, based on the above characteristics, can be considered as an educational and rehabilitation environment. As problems arise during the training of people with disabilities, changes in legal documents governing various aspects of the education and social protection of people with disabilities appear, it will be necessary to find adequate solutions to pressing issues of improving this environment. In addition, it is necessary to take into account the need to monitor long-term learning outcomes in the environment in question, focusing on its educational and rehabilitation functions, and based on the analysis of these results, adjust the environment.

The design of a digital educational environment in a university cannot take place without taking into account the main trends in the development of university educational technologies in general and, in particular, special education and rehabilitation technologies.

Under assistive technologies and accessibility technologies in the educational space, specialists consider a special combination of organizational structures and events, system tools and methods, general and private methods that optimally provide people with disabilities with the implementation and assimilation of educational programs in volume and quality, provided state educational standards. This totality also includes the formation of methods of cognition and activity at a general given level; personality habilitation in a specific integrated learning environment; the creation of a system of measures aimed at eliminating or possibly more fully compensating for the disabilities caused by health problems with persistent dysfunctions of the body, taking into account the restrictions in the educational environment on the duration of the training, the state of the material and technical base, qualifications of the person, intellectual, educational and rehabilitation potential of students and their special educational needs.

Assistive and accessibility technologies at the university must meet the requirement:

- compliance with the concept of continuous multilevel integrated education;

- inclusiveness and its inclusion in the established normative teaching practice of the university;

- integration into the national system of specialized vocational education;

- organic communication and complementarity of educational and rehabilitation technologies, i.e. ensuring the unity of educational and rehabilitation processes;

- the continuity of the scientific standards of traditional technologies with new technologies;

- understanding of special educational technologies as informational and intellectual. 
Technological and organizational approaches to providing the basis for vocational education of people with disabilities in the digital environment and its effective functioning require:

- preferences of intensive and high (including intellectual) learning technologies;

- the priority of information computer technologies that most fully comply with the requirements for special educational technologies formed above and are used to one degree or another in the learning process and the diverse technologies used in it.

The proper level of technical equipment of the educational process using the digital environment is achieved through the creation of special digital classrooms, classrooms, multifunctional digital modules, automated workstations for teachers and students with hearing impairment. This is due to the goal of creating the appropriate conditions necessary for the effective solution of the problem of creating a barrier-free information-educational environment (hereinafter - IEE) for this category of students. It is necessary to organize the educational space of a fundamentally new type as specialized system-integrated information complexes of educational resources and technologies.

Each complex is a functional module designed for specific goals and objectives. These modules are divided into the following groups:

Specialized digital classroom modules:

- Module "Lecture Audience";

- Module "Class";

- Module "Laboratory";

- Module "Fablab";

- Module "Consulting Center";

- Module "Audience of rehabilitation technologies".

Specialized digital multifunction modules:

- The module of distance learning technologies;

- The module of network integration in the information environment and the availability of educational space;

- A module for providing special support to the educational process;

- The module of technological support and support of the educational process;

- Module for supporting the educational process with technologies of total communication;

- Specialized automated workstations for disabled students (AWS);

- Specialized automated workstations of teachers;

- Specialized automated workplaces of personnel (ESS, specialists and mentors);

- Module of information and organizational support;

- The module of information and educational resources;

- Module for technical, technological and methodological support.

All digital audiences, classrooms and multifunctional modules must be integrated into a single local network with Internet access. Equipment for classrooms and modules should include technical training and rehabilitation facilities.

The selection and installation of these means is carried out taking into account their maximum compatibility with the sound equipment and individual and group technical rehabilitation tools used in the educational process (individual hearing systems and hearing aids, sound-amplifying equipment, group radio classes of FM bands, multi- media devices for supporting the educational process with sign language and creeping line, etc.). 
When designing and equipping classrooms and modules, the principle of software and hardware compatibility with other modules of the infrastructure, as well as with the information and educational environment of the university, is taken into account. In addition, it is necessary to ensure the continuity of educational technologies.

All this helps to minimize the hearing impairment of students and to fulfill the special educational needs of these students, providing them with: real access to relevant databases and educational materials in their workplace (and, if necessary, support in the form of sign language or traveling strings); in ensuring on-line and off-line participation of a disabled student in ongoing video conferences, both in local networks and in the global Internet network; in providing the ability to record on removable (external) storage media related to the educational process.

Information computer technologies in the educational and professional activities of people with hearing impairment in their special functional purpose are designed to provide a solution to a wide range of tasks, of which the most important are:

- physical access to personal computers as an instrument of professional (and educational) activity;

- the ability to effectively use intensive, high (including high intellectual) technologies of training and communication, including in those cases when access to them by conventional means is difficult due to specific life restrictions;

- the implementation of activities that are difficult to access for people with disabilities due to specific disabilities and which are not utilitarian functions for personal computers;

- a more complete correction and compensation (and/or substitution) of life limitation caused by loss or violation of the body's functions ("artificial ear" processors, programmable digital auditory systems, etc.) is possible.

Information computer technologies with the needs of people with disabilities can be conditionally divided into adaptive (actually adaptive, correlative adaptive, compensating adaptive) and integral interface.

The technologies of the first group are designed to alleviate the problems of the educational and professional activities of people with hearing impairment, and cannot, unfortunately, solve them completely. These technologies are implemented using personal computers with an exclusive configuration, original controllers, special software and a functionally adapted system of external devices.

Integrated interface technologies are aimed at a radical solution to the problem of rehabilitation and integration of hearing-impaired people. These technologies are implemented based on original processors, a system of controllers and special software manufactured with a high degree of individualization.

Despite the obvious diversity in the purpose, content, and implementation, an unconditional requirement to achieve real and long-term progress in the use of special education and rehabilitation technologies in the context of building a digital space is their system integration into a single technological base for training disabled people at a university. In this regard, the prerequisite for such integration is the universality of the university's technological base to persons with disabilities. Neglect of their special needs can lead to technological discrimination and, as a result, to a restriction in the educational and professional capacity of this category of citizens, especially in the context of the globalization of education and intellectual activity. A specially organized information and educational interface to the fullest extent meets the main goals of applying special technologies for teaching students with hearing impairment at a university - to serve as an instrument for integrating and improving the quality of education with all the variety of possible forms of organization of the educational process (general groups, special groups, special audiences, non-equipped audiences, lectures, seminars, etc.). 


\section{The results of the experiment on the use of digital technology in the teaching of academic disciplines at Bauman Moscow State Technical University}

In the 2017-2018 academic year, an experiment was carried out on teaching the discipline "Analytical Geometry" [14] using the information-educational environment NOMOTEX ${ }^{\dagger}$ $[7,8,9]$.

This system is a promising element of the new learning technology and contains great potential for the development and improvement of both the system itself and the construction of its educational content. Students with disabilities and hearing disabilities were included in the experiment. The educational process of these students has features that must be considered. To this end, methodological tasks have been developed at the State University of Management and Management of Science and Technology, which are used to minimize (and in some cases compensate) deviations from the standard level of students' cognitive qualities. When studying the educational process of people with disabilities (PD), it was proved that some of these deviations are universally recognized, that is, they can also occur in healthy students [4-6]. Therefore, many recommendations for the educational process are also generally valid and should be taken into account when designing electronic educational resources and teaching any students.

Consider from these positions an experimental online course (EOC) "Analytical Geometry" [14]. Minimum composition and technical implementation included:

- statement of the cognitive task;

- training material (mainly in video format);

- asks for the formation of competencies provided by educational programs;

- feedback;

- control and preservation of the results of students' activities;

- lists of used and recommended sources.

Organization of educational space. When lecturing, an electronic board, a monitor, and personal computers were used. Handout not used. When using personal computers, there is an interactive interaction between the teacher and students with the possibility of operational control, which is an indisputable advantage of the electronic learning method.

The content of the course coincides with the ordinary program of the academic discipline "Analytical geometry".

The structure of the distribution of educational material. The main structural unit of the course is a module that contains a thematically completed part of the training material (the number of modules is determined by the course program). A digital frame with cells ("drawers") has been created, which can be filled with various disciplines. The mainframe with the name of the part of the theme further has an extension of several frames. For example, there are frames "Definition", "Remarks", “Theorem", "Proof", valuable frames are "Criterion", "Engineering examples".

Interdisciplinary communications. The hierarchical construction of electronic content makes it possible to widely and widely reveal interdisciplinary connections, which allow

\footnotetext{
$\dagger$ IEE NOMOTEX - Information and educational environment for the mathematical training of engineers, created by BMSTU, Department "Computational Mathematics and Mathematical Physics" (FN-11) Performance Manager - Dimitrienko Y.I., Head of Department FN-11, Professor, Dr. Phys.-Math. Sci.
} 
not only more economical to build conclusions of mathematical and other concepts, but also motivate students (the need to study the discipline under study is recognized) to independently study the discipline.

The qualification requirements for teachers who develop and implement digital educational material, in addition to standard ones, include knowledge of the principles of universal design and the cognitive characteristics of those students for whom the electronic course is intended.

Qualification requirements for students from among PD include the skills specified in the IEE.

Special requirements for electronic educational courses should include knowledge about the functional and cognitive features of the contingent from among the disabled (hearing) and how to adapt the training material for them:

- to solve the problem of determining the current level of knowledge and the degree of readiness of the contingent to an adequate perception and understanding of educational material and the possibility of its adaptation, it is advisable to have cells with tasks of input control to establish the necessary level of knowledge in front of each major topic (module);

- to solve the problem of improving contextual recognition and understanding of educational material in the process of its presentation, it is imperative to provide for the possibility of the preliminary acquaintance of students with the terminological bases and relevant definitions, organized in the form of separate (cells) of audio and video files in auditory (sound: oral speech) and auditory-visual (video: text, articulation and/or gesture) forms;

- a prerequisite for the successful development of cognitive functions is to ensure highquality sound and pictures and comfortable conditions for their perception;

- to solve the problem of motivating the study of sections in a hierarchical network, it is necessary to indicate the paths (chains) of logical transitions from the current "picture" (frame) with the name of the topic or discipline to frames of the following topics (for example, the path from vector algebra to linear algebra, to analytic geometry, integral calculus, to physics, etc.);

- to solve the problem of visibility, it is advisable to actively use illustrative material with 3D animation;

- fixing the topic at the end (or its segment, block) requires separate cells ("boxes") with the results of the theory and the main practical applications;

- to solve the problem of responsibility formation, self-control of students should be practiced, for which purpose the appropriate cells should be included in the structure of electronic courses, the protocol of work with which excludes sending the results to the teacher without preliminary verification;

- to strengthen the cause-and-effect relationships when teaching people with disabilities, it is necessary to insert blocks with logical matrices of concept systems or a reformulation of the theorem with paragraphing and a subsequent proof plan, which is useful for the gradual formation of mental actions and concepts.

Currently, in the GUIMC BMSTU continues to work on the experimental use in the educational process of online courses [14] ("Updating knowledge in elementary mathematics" and "Effective ways of organizing independent work") and electronic manuals for students from PD [3].

This experiment showed that the experience of students themselves and other participants in the process (teachers, support staff) and their satisfaction with the quality of the services provided and the result plays a decisive role. In a digital educational environment, a student is more involved in his or her education. He has more complete 
access to educational content (subject to its universal design and the availability of special opportunities) and a closer connection can be established between all subjects. Digitalization allows students to interact effectively with subject-oriented websites, applications, and personal accounts, as well as with all participants in the educational process. With the help of these interactions, students with disabilities can present their status in the personal account of their teacher, tutor or fellow practitioner, thereby providing the ability to quickly discuss the results or provide assistance. A disabled student is allowed to become (more) an active participant in training in the form of classroom and independent work by partners understanding their personal preferences and experience, participating in creating such a learning environment that has active cooperation as its priority.

\section{Conclusion}

The challenges that professional education in a digital environment presents to disabled students in combination with an inclusive form of training predetermine the need for methodological, technical, technological and organizational support for the formation and development of such an environment. The development of this professional education is intended to significantly change the quality of the formation of professional competencies and the development of the ability of students with disabilities to use them in practical professional activities.

Engineering and technology are the main way to ensure equalization of conditions for (unlimited) access for people with hearing impairments to all types of activities: at home, in an educational institution, at the workplace, as well as in the social sphere. Digital technologies and appropriate training can give deaf and hard of hearing people the opportunity to experience the positive impact of equal access to teaching and communication tools on quality of life.

By the method of choosing the technological support for the formation of a digital environment, in the context of vocational education for people with hearing impairment, a specially organized information and educational interface should be considered that fully meets the main goals of the use of digital technologies for teaching students with hearing impairment in a university - to serve as an instrument integration and improving the quality of education with all the variety of possible forms of organization of the educational process (general groups, special groups, specialists Audiences, non-equipped audiences, lectures, seminars, etc.).

Considering higher education not only as a process of mastering a certain amount of knowledge in fixed subjects and disciplines but also as a process of forming ways of thinking and activity, it is shown that the active functioning and development of people with disabilities in a digital educational environment provides them with a high level of spiritual and moral and socio-psychological culture and contributes to their transfer from the category of passive and dependent consumers of educational services into active partners in educational and professional spheres.

The digital environment provides an opportunity for more free access to rehabilitation resources (assistive technologies, accessibility technologies and technical rehabilitation equipment (hereinafter - TRE) of a new generation with elements of artificial intelligence) and providing conditions for their deep system integration. Modern TRE and hearing systems (hearing aids) can be connected to various applications (including mobile ones), with the help of which they can track the characteristics of the sound environment and other behavioral information and can be adjusted in accordance with personal preferences user, in accordance with specific conditions in the educational space (classrooms, classes, 
laboratories, etc.). It is obvious that in the near future, new generation TRE, mobile and digital applications, and artificial intelligence will make it possible to organize a significant part of the processes of education and rehabilitation using a specially organized single interface.

The development and creation of models of the optimal infrastructure of the digital educational (and production) space and virtual collections of its main functional models will allow for situational modeling and expert assessment of the effectiveness of the use of digital adaptive resources, assistive technologies, intelligent TRE, and special TRED, in various activity and behavioral situations.

The development of effective technologies for creating a new generation of the adaptive electronic educational content of the new generation will ensure equalization of access and development of educational programs for students with disabilities in all forms of organizing an inclusive educational process.

An analysis of the learning experience of people with disabilities in an inclusive form in the digital educational environment of the university allows us to state the need to use large databases and knowledge bases. An analysis of the development of such databases by students will allow for an in-depth understanding and forecasting of the models of functioning and development of students with disabilities in the digital educational environment and help in making decisions regarding the methods and forms of training and support for each student and, thus, increase the efficiency training and rehabilitation.

To ensure the readiness of the faculty and staff to work effectively in a universal digital educational environment, it is necessary to develop educational programs of additional education (advanced training, internships and author's training courses, etc.) in the declared field in order to form professional interest and acquisition of necessary competencies.

The creation of a constantly online updated knowledge base of successful practices in the development and application of digital educational technologies, assistive technologies and accessibility technologies, intelligent TRE and special TRED, universal design technologies and the experience of their system integration into the digital educational environment will serve as a useful resource and practical guidance for experts, teachers, specialists, students, and all interested parties.

When launching the project of vocational education for people with disabilities in a digital environment, it is necessary to analyze in detail the problems of the participants in the educational process, identify the opportunities that the digital educational environment has in itself, and determine what special needs can be met with the help of new technologies within this environment. It is important not to create false or exaggerated expectations for all project entities; it is necessary to focus only on what can be accomplished at this stage and show the real prospects for the development of the project.

It is important to note that at the moment, none of the existing online platform forms for online courses in Russia (and, possibly, in the world) does have online courses that take into account the special educational needs of people with hearing impairments and other disabilities. This makes it possible for authors who have set themselves this noble and ambitious goal to become pioneers in this field and to declare their experience, at least, in the national scientific community.

\section{References}

1. N.A. Gorelov, O.N. Korableva, Development of the information society: digital economy: textbook for universities (series: Universities of Russia), Moscow, Publishing House "Yurayt" (2019)

2. E.M. Avlasovich, M.V. Vasyukova, Foreign experience in the development of digital educational technologies, Modern trends in the development of the education system, 
Collection of materials of the scientific and practical conference, pp. 12-14, Moscow (2019)

3. M.D. Konstantinov, D.S. Kuleshov, M.V. Mozgovoy, Interactive books for people with disabilities as a form of resource support for inclusive education, BMTSU, Electronic journal "Science and education", No. 07, pp. 296-307 (2016)

4. A.G. Stanevsky, Z.F. Stolyarova, Tasks of the correctional course of mathematics, Psychological Science and education, No. 2, pp. 48-58 (2012)

5. A.G. Stanevsky, Z.F. Stolyarova, Problems of adaptation of the main educational program at a university for people with hearing disabilities by ear, Electronic journal "Psychological science and education", Vol. 9, No. 1, pp. 23-37 (2017)

6. A.G. Stanevsky, Z.F. Stolyarova, The idea and justification of subject correctional courses", No. 1, pp. 46 - 55 (2012)

7. Y.I. Dimitrienko, E.A. Gubareva, T.V. Oblakova, A.A. Prozorovsky, Application of the NOMOTEX digital educational environment for training engineers in the course "Analytical Geometry”, Journal of Science, 10 (22), p. 7 (2018)

8. Y.I. Dimitrienko, E.A. Gubareva, D. A. Chebakov, Design of electronic interactive courses in the digital educational environment NOMOTEX, Journal of Science, 11 (35), p. 10 (2019)

9. Y.I. Dimitrienko, E.A. Gubareva, Neural Network Model of Mathematical Knowledge and Development of Information and Educational Environment for Mathematical Training Of Engineers, Journal of Physics: Conference Series, 012010 (2018)

10. Y.I. Dimitrienko, E.A. Gubareva, A new technology of mathematical training of engineering personnel, based on a neural network model of knowledge, Innovations in education, No. 11, pp. 129-140 (2017)

11. http://www.hearingreview.com/2015/08/amplification-new-information, access date 01/10/2020.

12. http://www.hearingreview.com/2015/08/cognitive-function-patient-landscape, access date $01 / 10 / 2020$.

13. http://www.hearingreview.com/2015/02/brainhearing-maximizing-hearing-listening/, access date.

14. https://online.bmstu.ru/courses/course-v1:BMSTU+1+e.g.2010t_1/abaut, access date 01/10/2020. 